\title{
Change in Choice-Related Response Modulation in Area MT during Learning of a Depth-Discrimination Task is Consistent with Task Learning
}

\author{
Takanori Uka, Ryo Sasaki, and Hironori Kumano \\ Department of Neurophysiology, Graduate School of Medicine, Juntendo University, Bunkyo, Tokyo 113-8421, Japan
}

What are the neural mechanisms underlying improvement in perceptual performance due to learning? A recent study using motiondirection discrimination suggested that perceptual learning is due to improvements in the "readout" of sensory signals in sensory-motor cortex and not to improvements in neural sensitivity of the sensory cortex. To test the generality of this hypothesis, we examined this in a similar but different task. We recorded from isolated neurons in the middle temporal (MT) area while monkeys were trained in a depth-discrimination task. Consistent with earlier reports using direction discrimination, we found no long-term improvement in MT neuron sensitivity to depth, although monkey performance improved over months with extensive training, even when taking out the effect of behavioral biases. We further addressed improvement in the readout of sensory signals by focusing on choice-related response modulation [choice probability (CP)]. CP increased with training, suggesting an improvement in the readout of sensory signals from MT. $\mathrm{CP}$, however, correlated more strongly with lapse rate than psychophysical threshold, suggesting that changes in readout may be restricted to early phases of learning. To test how behavioral learning, as well as the magnitude of $\mathrm{CP}$, transferred across visual fields, we measured CP variation in one hemifield after training monkeys on the depth-discrimination task in the opposite hemifield. CP was large from the beginning of training in the untrained hemifield, even though a small but significant improvement in sensitivity was observed behaviorally. Overall, our findings are consistent with the idea that increases in $\mathrm{CP}$ reflect task learning.

\section{Introduction}

Perceptual learning refers to the improvement in performance on a perceptual discrimination or detection task. Learning is often restricted to the exact stimuli and is often visual-fieldspecific (Fiorentini and Berardi, 1980; Schoups et al., 1995; but see Jeter et al., 2010; Zhang et al., 2010). This has led to the view that learning occurs in early visual areas containing neurons with small receptive fields (Karni and Sagi, 1991). Indeed, an increase in the sensitivity of sensory neurons has been found in areas V1 (Schoups et al., 2001) and V4 (Yang and Maunsell, 2004; Raiguel et al., 2006) after learning of an orientation-discrimination task. The change in sensitivity, however, is minimal and is unlikely to explain the magnitude of learning observed behaviorally.

Using a motion-direction discrimination task, Law and Gold (2008) showed that perceptual learning can be explained

Received Aug. 21, 2010; revised July 31, 2012; accepted Aug. 4, 2012.

Author contributions: T.U. designed research; T.U., R.S., and H.K. performed research; T.U., R.S., and H.K. analyzed data; T.U., R.S., and H.K. wrote the paper.

This work was supported by Grants-in-Aid for Scientific Research on Priority Areas (System study on higher-order brain function) from the Ministry of Education, Culture, Sports, Science and Technology of Japan (MEXT; Grant 17022034), and the Nakajima Foundation to T.U. R.S. and H.K. were supported by a High Technology Research Center Grant from MEXT. We thank Sanae Hosotani for technical and surgical assistance.

Correspondence should be addressed to Takanori Uka, Department of Neurophysiology, Graduate School of Medicine, Juntendo University, 2-1-1 Hongo, Bunkyo, Tokyo 113-8421, Japan. E-mail: uka@juntendo.ac.jp.

R. Sasaki's present address: Department of Brain and Cognitive Sciences, Center for Visual Science, University of Rochester, Rochester, NY 14627.

DOI:10.1523/JNEUROSCI.4406-10.2012

Copyright $\odot 2012$ the authors $\quad 0270-6474 / 12 / 3213689-12 \$ 15.00 / 0$ by an improvement in the "readout" of sensory signals (Mollon and Danilova, 1996; Dosher and Lu, 1999; Petrov et al., 2005; Michel and Jacobs, 2008; Jacobs, 2009) in a sensorymotor area. They recorded simultaneously from the middle temporal (MT) area and the lateral intraparietal (LIP) area in monkeys and found that the rate of build-up activity in LIP increased with learning, whereas sensitivities of MT neurons to motion did not change throughout the course of training. They also showed that choice-related response modulation [choice probability (CP)] increased for MT neurons that were sensitive to changes in motion coherence. Law and Gold (2008) suggested that, due to learning, the monkeys became capable of selectively reading out optimal sensory information from neurons with relevant information, and thus behavioral performance improved.

In this study, we addressed the generality of this idea by testing monkeys in a similar but different discrimination task. We recorded from isolated neurons in area MT while monkeys learned a depth-discrimination task. It is fairly well established that MT contains neurons that are selective for binocular disparity (Maunsell and Van Essen, 1983; DeAngelis and Uka, 2003) and that these neurons are relevant for execution of the depth-discrimination task (DeAngelis et al., 1998; Uka and DeAngelis, 2003, 2004, 2006; Chowdhury and DeAngelis, 2008).

Consistent with Law and Gold (2008), we found no long-term improvement in the sensitivities of MT neurons to depth, although monkey performance improved with extensive training. 
We also measured $\mathrm{CP}$ and found an increase with training. $\mathrm{CP}$, however, correlated more strongly with lapse rate than psychophysical threshold. To test how behavioral sensitivity, as well as the magnitude of $\mathrm{CP}$, transferred across visual fields, we trained monkeys on the depth-discrimination task at a fixed location and subsequently recorded activity from the hemisphere ipsilateral to the trained location while monkeys learned to perform the task in the opposite hemifield. The monkeys' behavior quickly improved in the untrained hemifield. CP, however, was sufficiently large from the beginning of training in the untrained hemifield and did not increase across training sessions. The results are consistent with the idea that increases in $\mathrm{CP}$ reflect task learning.

\section{Materials and Methods}

The general experimental procedures for singleunit recording from area MT of awake monkeys were described in detail previously (Uka and DeAngelis, 2003). All animal care, training, and experimental procedures were in accordance with the National Institutes of Health guidelines and were approved by the Juntendo University Animal Care and Use Committee.

\section{Subjects and surgery}

Experiments were performed using four (3 male and 1 female) Japanese monkeys (Macaca fuscata) weighing 4-9 kg. A post for head restraint and a recording chamber were chronically implanted in each monkey. We implanted scleral search coils into both eyes to monitor eye movements (Judge et al., 1980). The recording chamber was mounted over the occipital cortex $\sim 17 \mathrm{~mm}$ lateral and $14 \mathrm{~mm}$ dorsal to the occipital ridge at an angle of $25^{\circ}$ above the horizontal, such that area MT was accessed after passing through striate cortex and extrastriate visual areas in the lunate sulcus.

\section{Visual stimuli}

The monkeys sat in a primate chair and faced a flat-screen 22-inch CRT color monitor (HM204DA; Iiyama) placed at a viewing distance of $57 \mathrm{~cm}$. The display subtended a visual angle of $40^{\circ} \times 30^{\circ}$, had a resolution of $1280 \times$ 960 pixels, and was refreshed at $100 \mathrm{~Hz}$. Visual stimuli were generated using a dual CPU workstation running Windows XP. Random-dot stimuli were programmed in Microsoft Visual $\mathrm{C}++$ using the OpenGL libraries and were displayed by an OpenGL accelerator board with quad-buffered stereo support (Quadro4 980 XGL; NVIDIA). Each random-dot stereogram (RDS) was presented within a circular aperture. Dot density was 64 dots per square degree per second, with each dot subtending $\sim 0.1^{\circ}$.

The starting position of each dot within the aperture was newly randomized for each trial. Precise disparities and smooth motion were achieved by plotting dots with subpixel resolution with the hardware anti-aliasing capabilities of the OpenGL accelerator board.

$\mathrm{B}$
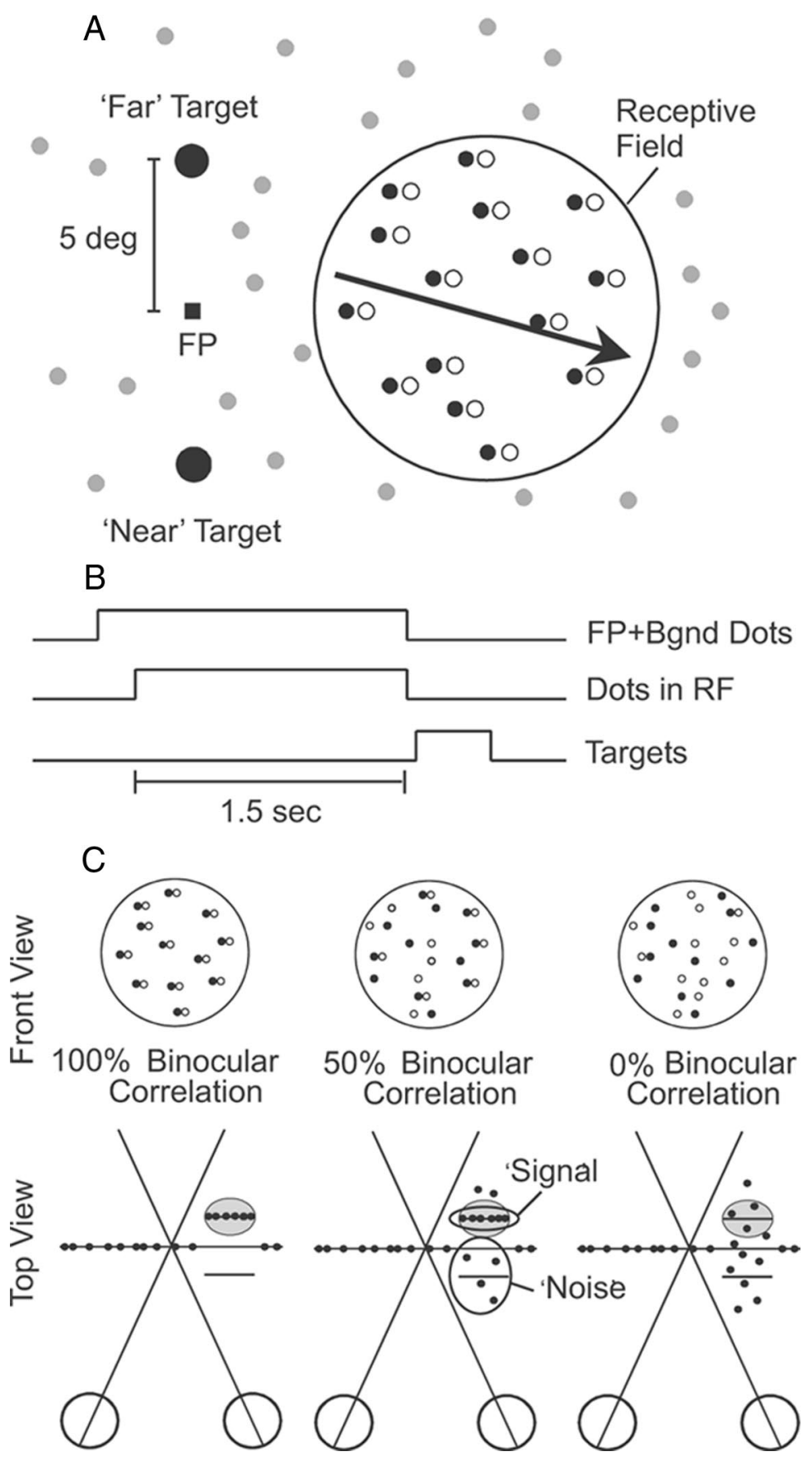

Figure 1. Depth discrimination task. $\boldsymbol{A}, \mathrm{A}$ random-dot stereogram was presented within a circular aperture slightly larger than the neuron's receptive field (RF), and dots moved at the neuron's preferred velocity (arrow). Filled and open dots represent left- and right-half images, respectively. Regions of the $40^{\circ} \times 30^{\circ}$ screen outside the receptive field were filled with flickering zero-disparity background dots (gray). Saccade targets were located $5^{\circ}$ above and below the fixation point, corresponding to "far" and "near" choices, respectively. $\boldsymbol{B}$, Time course of a discrimination trial. The fixation point (FP) first appeared along with the background (Bgnd) dots. After the random-dot stereogram was presented for $1.5 \mathrm{~s}$, the fixation point and dots were extinguished, and two choice targets appeared. Monkeys reported the depth of the stimulus by making a saccade to one of the two targets. C, Manipulation of task difficulty. The depth signal strength was adjusted by varying the binocular correlation. At $100 \%$ binocular correlation (left), all dots within the receptive field were presented at either the neuron's preferred disparity (i.e., short horizontal line inside gray oval) or the disparity that elicited a minimal response (i.e., null disparity). At 50\% binocular correlation (middle), half of the dots had random disparities, thus forming a 3D cloud of disparity noise. At 0\% correlation (right), all dots were assigned random disparities, making the stimulus ambiguous.

Stereoscopic images were displayed by presenting the left and right half-images alternately at a refresh rate of $100 \mathrm{~Hz}(50 \mathrm{~Hz}$ for each eye). The monkeys viewed the display through a pair of ferro-electric liquid crystal shutters (DisplayTech) that were synchronized to the video refresh such that one shutter was closed while the other was open. Ghosting 
A

Monkey G
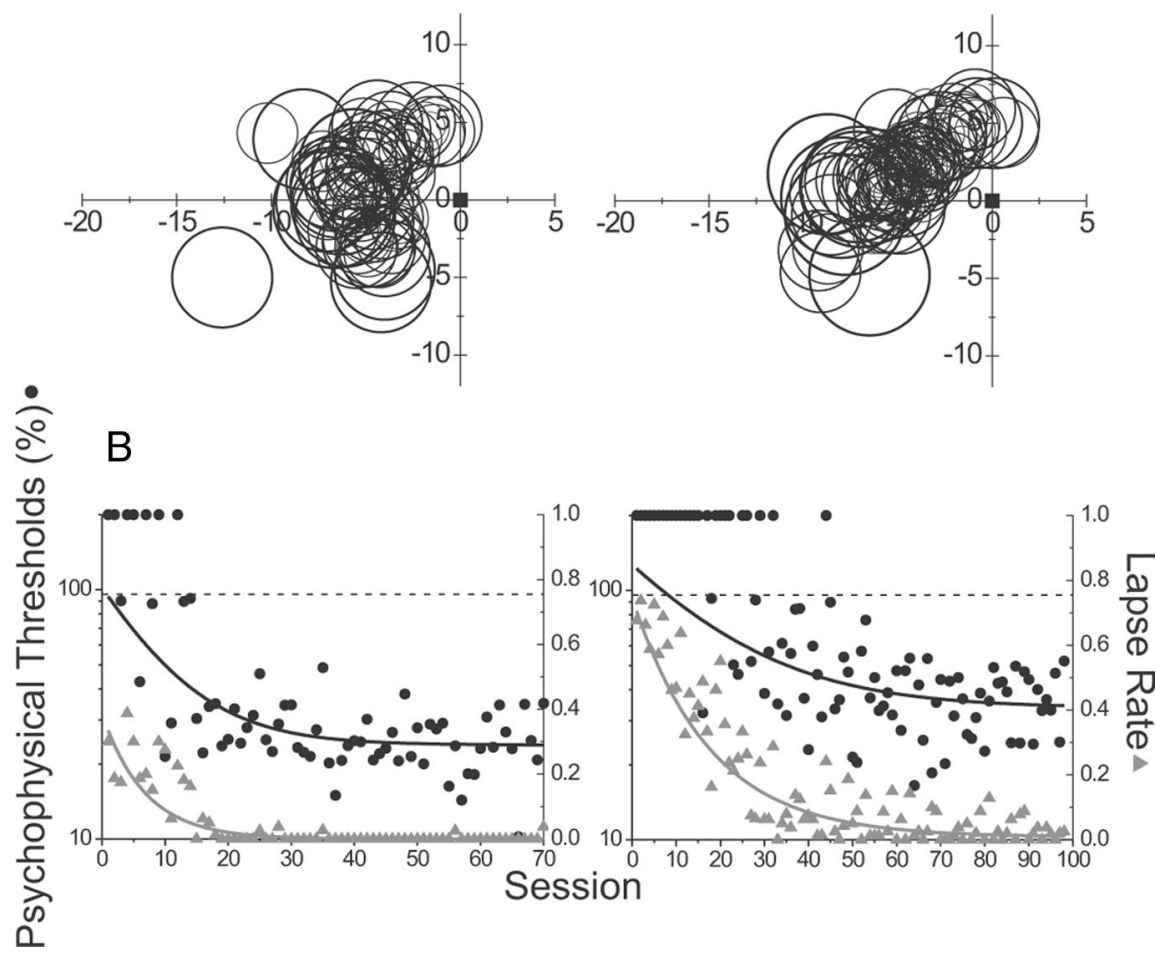

Figure 2. Analysis of psychophysical performance. A, Stimulus location for all recording sessions in the two monkeys. Circles correspond to the aperture locations where random-dot stimuli were presented. Aperture locations were determined from receptive field maps and area summation curves for each neuron. $\boldsymbol{B}$, Long-term improvement in psychophysical performance. Lapse rate (error rate at $96 \%$ correlation, triangles) and psychophysical thresholds (circles) are plotted as a function of daily recording sessions separately for the two monkeys. Solid lines denote exponential fits excluding thresholds $>96 \%$. Thresholds $>96 \%$ are plotted as 200\%. Dotted line indicates $96 \%$.

effects were minimized by presenting red dots on a black background, as the decay of the red phosphor is much faster than that of green or blue phosphor.

\section{Pretraining}

Behavioral tasks and data acquisition were controlled by a commercially available software package (TEMPONET; Reflective Computing) and on-line data analyses were done using MATLAB (MathWorks). The positions of both eyes were sampled at $1 \mathrm{kHz}$ and stored at $250 \mathrm{~Hz}$. Monkeys were first trained on a fixation task in which they were required to fixate on a yellow spot $\left(0.15^{\circ} \times 0.15^{\circ}\right)$ within a $2.0^{\circ} \times 2.0^{\circ}$ electronic window. Monkeys received a water or juice reward for maintaining fixation throughout a $1.5 \mathrm{~s}$ trial. When the monkey's conjugate eye position left the fixation window prematurely, the trial was aborted.

After fixation training, monkeys were subsequently trained on a direction-discrimination task to acclimate to a two-alternative forced-choice task. A random-dot stimulus that moved either upward or downward was presented on each trial, and the monkeys were required to report whether the dots moved up or down by making a saccade to one of two targets (located $5^{\circ}$ below and above the fixation point, respectively) that appeared $200 \mathrm{~ms}$ after stimulus offset. The saccade had to be made to one of the two targets within $1 \mathrm{~s}$ after their appearance, and the saccade endpoint had to remain within $2.5^{\circ}$ of the target for at least $150 \mathrm{~ms}$ to be considered a valid choice. Correct responses were rewarded with a drop of water or juice. The location of the aperture was $10^{\circ}$ to the left of the fixation point. The aperture size was $10^{\circ}$ and dot speed was $10^{\circ} \%$ at zero disparity. Discrimination training began with $100 \%$ motion coherence trials, and lower coherences were gradually introduced after the monkey reached at least $75 \%$ correct responses. The range of coherence levels was then pushed downward gradually over many weeks of training until the monkey's performance reached a plateau and did not improve further.

\section{Electrophysiological recordings}

A tungsten microelectrode (tip diameter 7-15 $\mu \mathrm{m}$, impedance $0.5-2 \mathrm{M} \Omega$ at $1 \mathrm{kHz}$; Frederick Haer) was advanced into the cortex through a transdural guide tube using a micromanipulator (MO-951; Narishige) mounted on the recording chamber. Single neurons were isolated using a conventional amplifier with a bandpass filter $(500-5000 \mathrm{~Hz})$ and a window discriminator (Bak Electronics). Times of occurrence of action potentials and trial events were stored to disk with $1 \mathrm{~ms}$ resolution. Area MT was recognized based on interpreting the pattern of gray matter and white matter encountered during electrode penetrations and on the physiological response properties of both single neurons and multiunit clusters.

\section{Depth-discrimination task and experimental protocol}

After training on the direction-discrimination task was completed, two of the four monkeys (monkey $\mathrm{G}$ and monkey $\mathrm{P}$ ) were trained on the depth-discrimination task (Fig. 1) while we recorded activity from MT neurons. In the depth-discrimination task, the monkeys were required to report whether dots within an RDS were near (crossed) or far (uncrossed) compared with the plane of fixation by making a saccade to the bottom or top target, respectively. All dots within the RDS moved coherently (100\% motion coherence) at a velocity tailored to each MT neuron. The disparity signal was titrated by manipulating the percentage of binocularly correlated dots in the RDS. Correlated (signal) dots were assigned one of two fixed disparities (crossed vs uncrossed) during each trial, and the remaining (noise) dots were assigned random disparities within the range from $-2^{\circ}$ to $2^{\circ}$ (Fig. 1C). Flickering background dots were presented at zero disparity to help anchor the monkey's vergence posture (Fig. $1 \mathrm{~A}$, gray dots).

The experimental procedures were as follows. After isolating an MT neuron, we crudely mapped the receptive field and estimated the neuron's preferred direction, speed, and horizontal disparity. Next, we quantitatively measured the direction, speed, size, and horizontal disparity tuning of each neuron. All tuning measurements were done in blocks of randomly interleaved trials, and responses were averaged across three to five repetitions of each distinct stimulus. Preferred values were determined online by visual inspection of the tuning curves. For the disparitytuning curves, the trough of the curve (null disparity) was also determined.

Following these tests, we recorded while the monkey performed the depth-discrimination task. Binocular correlation and stimulus disparity (preferred and null) were varied in blocks of randomly interleaved trials. The binocular correlation was always $0,3,6,12,24,48$, and $96 \%$. Whenever possible, data were collected for 40 repetitions of each unique stimulus condition, a total of 560 trials per daily session. If the monkey ceased to perform or neuronal isolation was lost prematurely, experiments were concluded for that particular day. Data were acquired for at least 10 repetitions (i.e., 140 trials). The average number of repetitions was $36.4 \pm 7.59$ (mean \pm SD; median: 40 ), and the average number of total trials was $510 \pm 106$ (median: 560).

To test for transfer effects across the visual field, we trained the remaining two monkeys (monkey $\mathrm{M}$ and monkey $\mathrm{K}$ ) on the depth- 
Table 1. Time constant of psychophysical parameters

\begin{tabular}{|c|c|c|c|c|c|c|c|c|c|}
\hline \multicolumn{5}{|c|}{ Initial training } & \multicolumn{5}{|l|}{ Transfer } \\
\hline \multicolumn{3}{|c|}{ Exponential fit } & \multicolumn{2}{|c|}{ Nonparametric } & \multicolumn{3}{|c|}{ Exponential fit } & \multicolumn{2}{|c|}{ Nonparametric } \\
\hline \multirow[b]{2}{*}{ Lapse rate } & \multicolumn{2}{|c|}{ Psychophysical threshold } & \multirow[b]{2}{*}{ Lapse rate } & \multirow[b]{2}{*}{ Psychophysical threshold } & \multirow[b]{2}{*}{ Lapse rate } & \multicolumn{2}{|c|}{ Psychophysical threshold } & \multirow[b]{2}{*}{ Lapse rate } & \multirow[b]{2}{*}{ Psychophysical threshold } \\
\hline & Excluding $>96 \%$ & Including $>96 \%$ & & & & Excluding $>96 \%$ & Including $>96 \%$ & & \\
\hline 6.8 & 9.1 & 8.9 & 19 & 31 & NA & NA & NA & NA & NA \\
\hline 17.6 & 20.1 & 22.1 & 54 & 71 & NA & NA & NA & NA & NA \\
\hline 37.6 & 165.2 & 56.8 & 151 & 160 & 1.4 & 3.3 & 1.4 & 30 & 30 \\
\hline 8.0 & 9.1 & 7.8 & 36 & 50 & 2.7 & 4.1 & 3.3 & 16 & 14 \\
\hline 7.5 & 26.1 & 12.1 & 19 & 30 & 1.3 & 0.70 & 2.2 & 3 & 10 \\
\hline
\end{tabular}

discrimination task at a fixed location without neural recording following completion of training on the direction-discrimination task. The stimulus location was $5.4^{\circ}$ to the right (for monkey $\mathrm{M}$ ) or left (for monkey $\mathrm{K}$ ) of fixation, and the aperture size was fixed at $7^{\circ}$; these were the median eccentricity and receptive field size of the recorded neurons from monkey G and monkey P. Each day, groups of motion direction, speed, and horizontal disparities were randomly chosen from parameters used in recording sessions from monkey G and monkey P. After extensively training the monkeys, we subsequently recorded from area MT in the ipsilateral hemisphere, so that the receptive field of the recorded neuron would be on the side opposite the trained hemifield. During recording, the monkeys were trained on the depth discrimination task at the receptive field location, which was an untrained location for the monkeys.

\section{Data analysis}

Neural responses were calculated from the firing rate during the $1.5 \mathrm{~s}$ stimulus presentation period. Spontaneous activity was calculated using the response to a blank screen.

\section{Calculation of psychophysical metrics}

Lapse rate and psychophysical thresholds. Monkey performance was evaluated by calculating lapse rates (Law and Gold, 2008) and psychophysical thresholds (Britten et al., 1992; Uka and DeAngelis, 2003). Lapse rates were determined as the monkeys' error rate at $96 \%$ correlation, the highest correlation level used in our study. Psychophysical thresholds were determined by plotting the monkeys' correct responses as a function of binocular correlation, which were then fit with a cumulative Weibull function given by the following:

$$
p=(1-\lambda)-(0.5-\lambda) e^{-(c / \alpha)^{\beta}},
$$

where $c$ is the binocular correlation of the stimulus, $p$ is the proportion of correct responses, $\alpha$ defines the threshold at $82 \%$ correct, $\beta$ gives the slope of the curve, and $\lambda$ is associative (high correlation) error. Here, $\lambda$ does not equal lapse rate described above. Learning rates were determined by fitting an exponential function to the daily lapse rate and psychophysical threshold curves. Because thresholds larger than $96 \%$ are unreliable, we estimated the time constant in two ways, one excluding thresholds $>96 \%$ and another using all data and clipping thresholds larger than $200 \%$ at $200 \%$. We further determined a nonparametric time constant. We determined the median lapse rate and threshold of the last 10 sessions in each monkey.
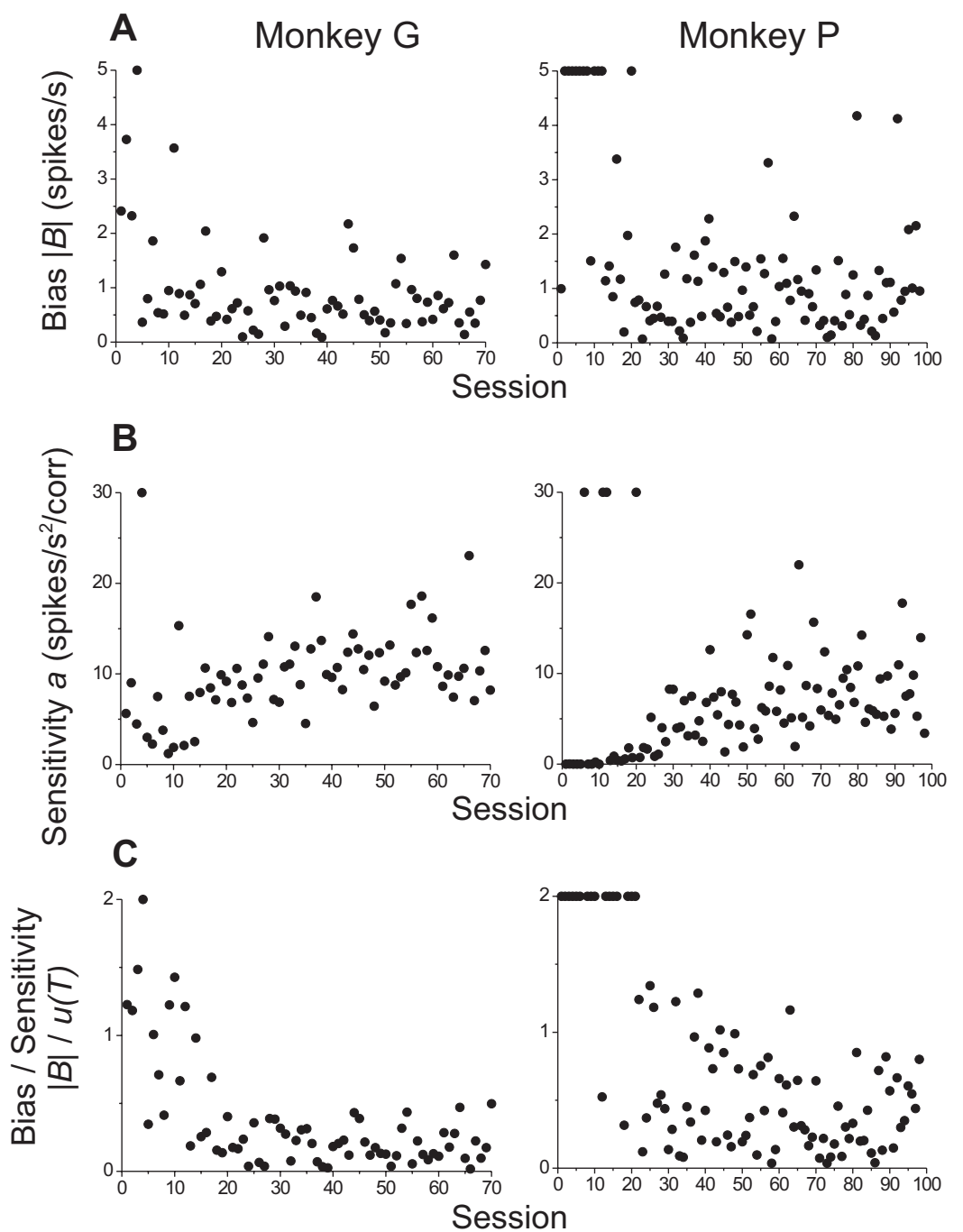

Figure 3. Bias analysis. $A$, The amount of bias is plotted as a function of recording sessions separately for the two monkeys. Biases larger than 5 are plotted as $5 . \boldsymbol{B}$, Sensitivities are plotted as a function of recording sessions separately for the two monkeys. Sensitivities larger than 30 are plotted as 30 . C, The relative contributions of biases compared with sensory signals are plotted as a function of recording sessions separately for the two monkeys. Values larger than 2 are plotted as 2.

We then determined the session where lapse rates or thresholds were lower than the median lapse rate and threshold of the last 10 sessions in three sequential sessions.

Sequential bias. Behavioral biases can interfere with calculation of sensitivity especially early during learning. We attempted to analyze and eliminate biases dependent on the sequence of previous trials (sequential bias). Here, we calculated sequential biases using the Weiner kernel analysis (Gold et al., 2008). Following Gold et al. (2008), we first defined choice residual (i.e., bias) as the difference between the predicted prob- 


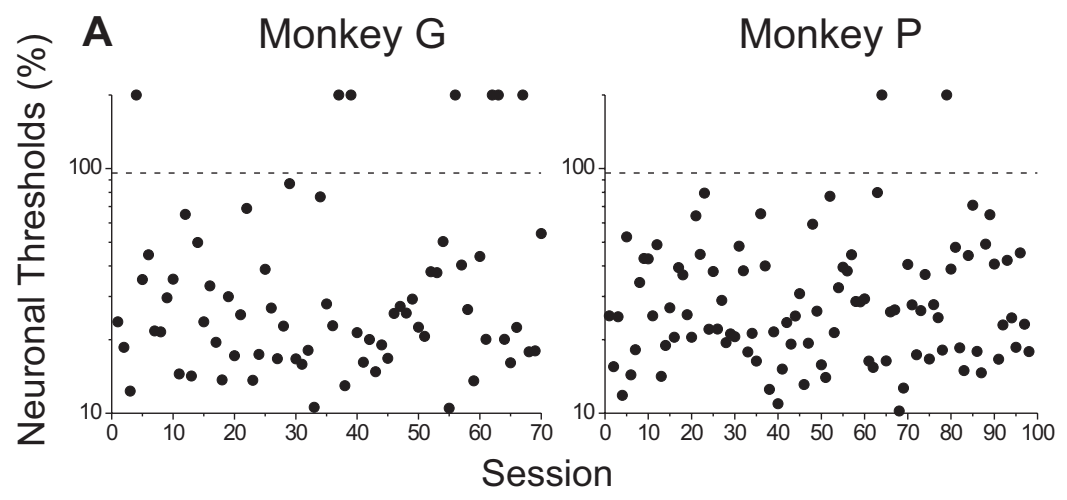

B
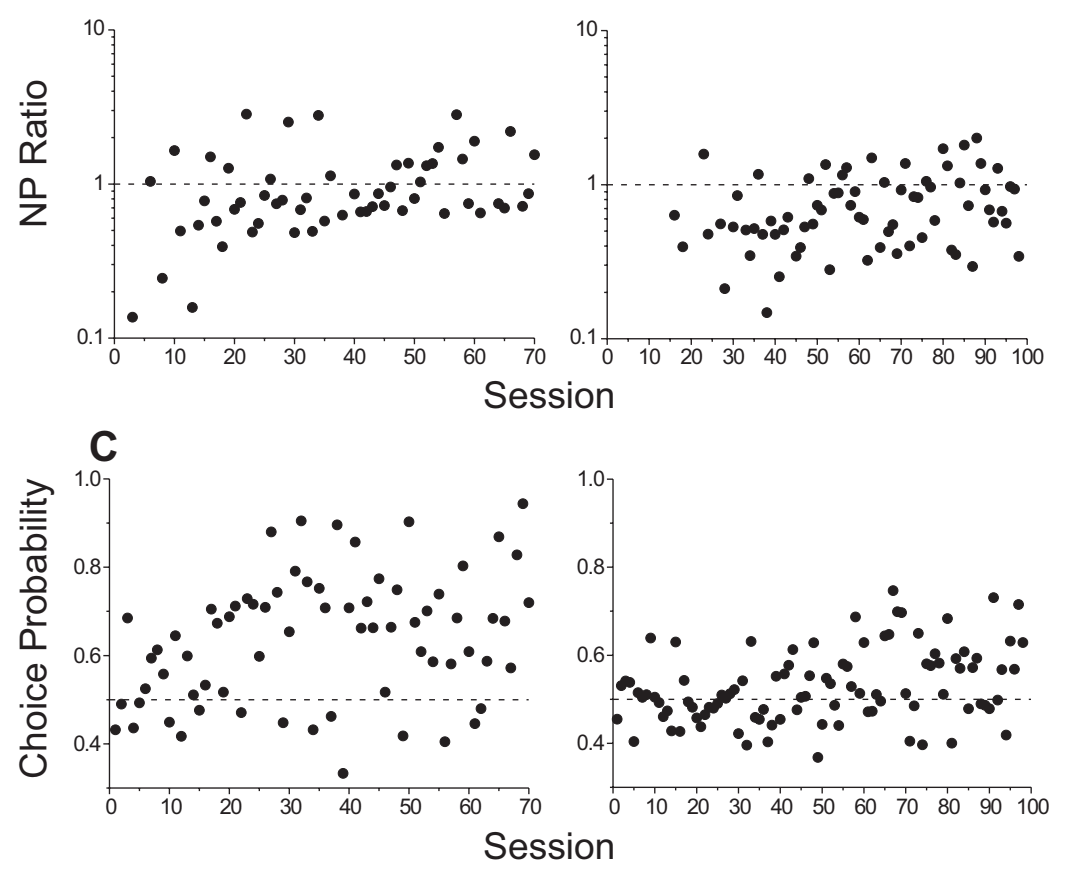

Figure 4. No long-term improvement in neuronal threshold. $\boldsymbol{A}$, Neuronal thresholds are plotted as a function of recording sessions separately for the two monkeys. Thresholds larger than $96 \%$ are plotted as $200 \%$. Dotted line indicates $96 \%$. B, NP ratios are plotted as a function of recording sessions separately for the two monkeys. NP ratios were calculated by dividing neuronal thresholds by psychophysical thresholds. Sessions with thresholds $>96 \%$ were excluded. C, Long-term increase in CP. CPs are plotted as a function of recording sessions separately for the two monkeys. Dotted line indicates 0.5 .

ability of making a preferred choice for a given stimulus and the actual binary choice. The predicted probability of making a preferred choice for a given stimulus ranged from 0 for null choices to 1 for preferred choices, determined from the fits to the psychometric function and taking into account the sign of the stimulus. The actual binary choice was also defined to be 1 for preferred choices and 0 for null choices. Because the predicted probability of making a preferred choice ranged from 0 to 1 , and the actual binary choice was either 0 or 1 , choice residual ranged from -1 when the predicted probability of making a preferred choice was 1 but the actual choice was the null choice $(0)$ to 1 when the predicted probability of making a preferred choice was 0 but the actual choice was the preferred choice (1) (Gold et al., 2008, their Fig. 2 B).

Next, the dependence of choice residual on previous trials was determined using the Wiener kernel analysis. Using the Wiener-Hopf equation, the Weiner expansion of the function relating past choices to the sequence of choice residuals were calculated as $W_{\mathrm{opt}}=R_{\mathrm{XX}}^{-1} R_{\mathrm{yX}}$, where $R_{\mathrm{XX}}$ is the autocorrelation matrix of the binary choice sequence and $R_{\mathrm{yX}}$ is the cross-correlation matrix of the binary choice and choice residual sequence.

Since this matrix captures most of the structure underlying bias, including idiosyncratic structures in the data, we estimated the gen- eral strategy underlying bias by fitting the first-order kernel with exponential functions, assuming that the dependence of bias on previous trials decay with time. As in Gold et al. (2008), the first-order kernel with positive lags (thus only causal relations were considered) was fit with two doubleexponential functions to calculate two filters, one for correct choices and another for errors: filters were calculated separately for correct and incorrect choices to capture the effect of reward on bias (i.e., such as winstay/lose-alternate strategies). Trial-by-trial choice residuals were then estimated (sequential biases, $S_{t}$, described below) by convolving the sequence of binary choices with the two filters followed by summation.

To calculate sensitivity taking out the effect of bias, the estimated choice residuals were then incorporated in the psychometric function and choice data were newly fit with the following function:

$$
\begin{gathered}
p=\lambda+(0.5-\lambda) \\
\times\left[1+\operatorname{erf}\left(\frac{\mu(T)+B}{\sqrt{2 \phi\left(2 r_{0}+a c^{m}\right) T}}\right)\right], \\
\mu(T)=a c^{m} \\
B=\beta_{s}+\beta_{w k} \times S_{t}
\end{gathered}
$$

where free parameters $\lambda$ is associative error, $a$ is sensitivity, $\beta_{s}$ is static bias, and $\beta_{\mathrm{wk}}$ is the weight for sequential bias. $T$ is $1.5 \mathrm{~s}, c$ is binocular correlation, and $S_{t}$ is sequential bias on trial $t$. The remaining parameters were set to values used previously ( $m=1.25, \varphi=0.3, r_{0}=10$ spikes/s) (Eckhoff et al., 2008). The amount of bias for each session was quantified as the mean absolute $B$, which incorporates both static and sequential biases.

\section{Calculation of neuronal thresholds}

To characterize the sensitivity of MT neurons in the depth-discrimination task, we used receiver operating characteristic (ROC) analysis to calculate neuronal thresholds based on the "anti-neuron" formulation (Britten et al., 1992; Uka and DeAngelis, 2003). An ROC curve was calculated from the response distributions to the preferred and null disparities at each correlation level. The area under the ROC curve was taken as the ability of an ideal observer to discriminate between the two disparities based solely on the responses of the recorded neuron (and an assumed anti-neuron with opposite preferred and null disparities). A plot of the ROC area as a function of binocular correlation defines the neurometric function, which was fit with the cumulative Weibull function given above, with associative error fixed at zero.

\section{Calculation of choice probability}

We quantified the relationship between MT responses and the animals' choices by computing CP using ROC analysis (Britten et al., 1996; Uka and DeAngelis, 2004). At each disparity and binocular correlation level, MT neuron responses were sorted into two groups based on the choice that the animal made at the end of each trial (i.e., preferred choices vs null choices). An ROC curve was calculated from these response distributions, and the area under the ROC curve gave the CP for that disparity/ binocular correlation combination. To arrive at a single grand CP for each neuron, responses were normalized (using $z$-scores) separately for 
each disparity/binocular correlation combination, and the normalized responses were then combined across stimulus conditions into a single pair of distributions for preferred and null choices. Trials from disparity/binocular correlation levels in which the monkeys preferred one target $>75 \%$ of the time were excluded (Uka and DeAngelis, 2004). ROC analysis on this pair of distributions yielded the grand $\mathrm{CP}$.

\section{Measurement of symmetry of}

disparity-tuning curves

To measure the symmetry of each disparitytuning curve around zero disparity, the curve was fit with a Gabor function that was constrained to have its Gaussian envelope centered at zero disparity:

$$
\begin{aligned}
R(d)=R_{0}+A \times e^{-0.5 \frac{(d)^{2}}{\sigma^{2}}} & \\
& \times \cos (2 \pi f(d)+\Phi),
\end{aligned}
$$

where $d$ is the stimulus disparity, $R_{0}$ is the baseline response level, $A$ is the amplitude, $\sigma$ is the standard deviation of the Gaussian, $f$ is the frequency of the sinusoid, and $\Phi$ is the phase of the sinusoid. $\Phi$ was then wrapped into the range from 0 to $\pi / 2\left(90^{\circ}\right)$, and this was used as an estimate of the symmetry of the disparity-tuning curve around zero. Values close to 0 indicate even symmetry, whereas values close to $\pi / 2$ indicate odd symmetry.

\section{Results}

In this study, we trained monkeys on a depth-discrimination task while we recorded activity from neurons in area MT. As we were interested in how neural characteristics changed due to learning, ideally we should have recorded from the same neuron throughout the course of learning. Because this was not possible, we recorded from different neurons each day and investigated how characteristics among neurons changed due to learning.

Perceptual learning is known to often be specific for visual features such as retinotopic location (Schoups et al., 1995). We therefore aimed to record from neurons with similar receptive field locations by penetrating the electrode each day through the same transdural guide tube. Figure $2 A$ shows receptive field locations, or stimulus locations used for training, for all neurons recorded from the two monkeys that were recorded from the beginning of training. Although stimulus location varied, eccentricities were restricted. Eccentricities ranged from $3.33^{\circ}$ to $13.6^{\circ}\left(\right.$ median $\left.=5.4^{\circ}\right)$, with the majority $(81.9 \%)$ within the range of $4.0^{\circ}$ to $7.0^{\circ}$. We recorded from all MT neurons that we could isolate, including several neurons with very weak or no apparent disparity tuning. This was done so that we would not bias our sampling.

\section{A Monkey M}
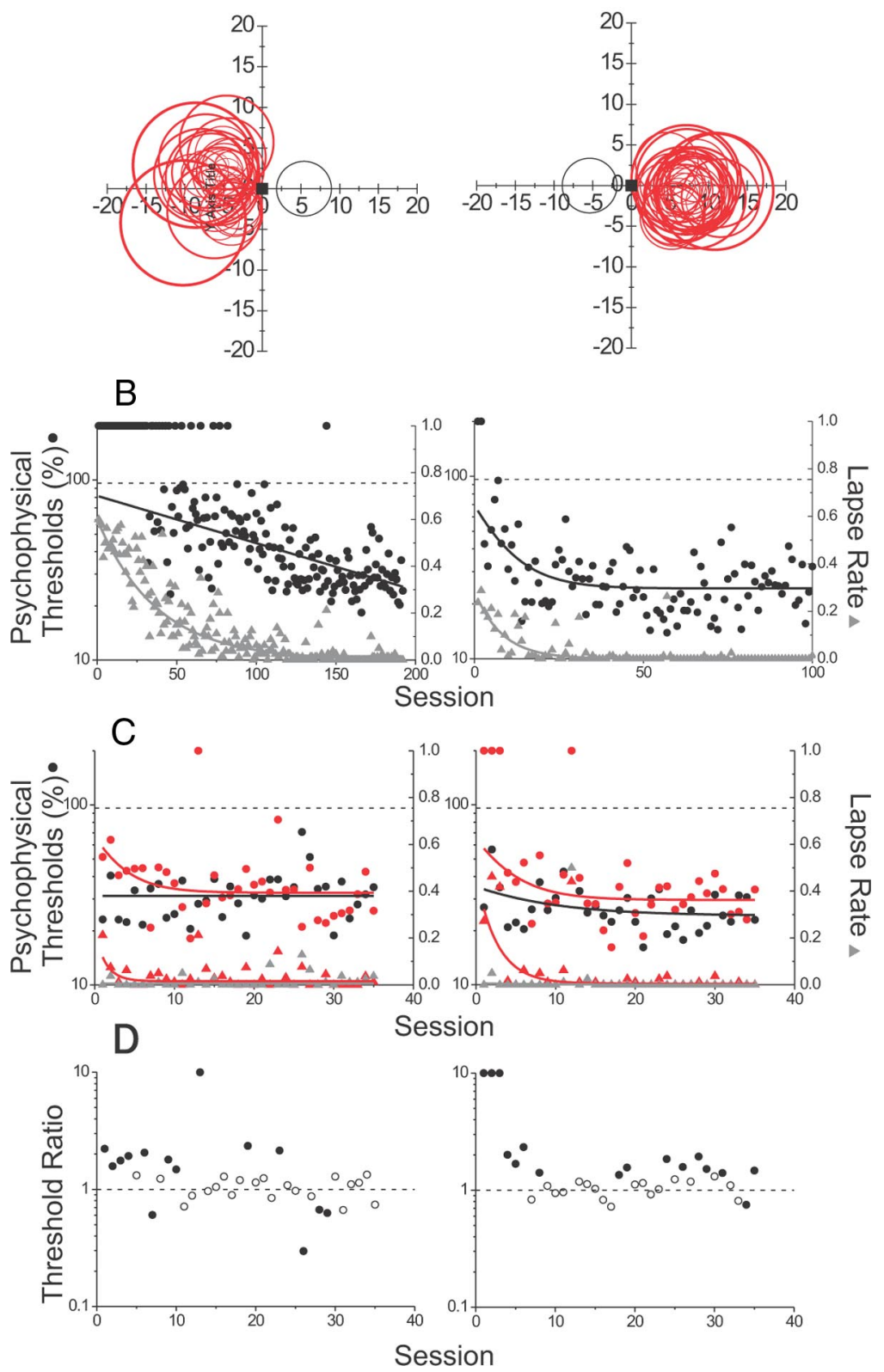

Figure 5. Transfer across visual field. $A$, Stimulus location for all recording sessions in monkey $M$ and monkey K. The black circle corresponds to the location where random-dot stimuli were presented during training. After training at the location shown with the black circle, recordings commenced. Red circles correspond to the aperture locations where random-dot stimuli were presented during recording. Aperture locations were determined from receptive field maps and area summation curves for each neuron. $\boldsymbol{B}$, Long-term learning at the trained location. Lapse rate (triangles) and psychophysical thresholds (circles) are plotted as a function of sessions for training at the fixed location shown with the black circle in $A$. Solid lines denote exponential fits excluding thresholds $>96 \%$. Thresholds larger than $96 \%$ are plotted as 200\%. Dotted line indicates $96 \%$. C, Long-term learning at new locations after learning in the opposite visual field. Red triangles and circles show lapse rate and psychophysical thresholds, respectively, at new locations determined from the receptive field maps and area summation curves of the neuron under study. Black triangles and circles correspond to lapse rate and psychophysical thresholds, respectively, at the trained location shown with the black circle in $A$ using the same stimulus parameters other than location and aperture size. These data were obtained the day after recording. Solid lines denote exponential fits excluding thresholds $>96 \%$. Thresholds larger than $96 \%$ are plotted as $200 \%$. Dotted line indicates $96 \%$. D, Psychophysical threshold ratios (untrained/trained) are plotted as a function of recording sessions. Session where either threshold was $>96 \%$ is plotted at 10 . Sessions where both thresholds were larger than $96 \%$ were excluded. Filled circles denote sessions with thresholds that were significantly different between trained and untrained hemifields (Bootstrap, $p<0.05$ ).

Thus, stimulus parameters other than location varied widely among daily sessions. A total of 70 and 98 recording sessions, consisting of 37,854 and 47,778 total trials, were performed for monkey $\mathrm{G}$ and monkey $\mathrm{P}$, respectively. 

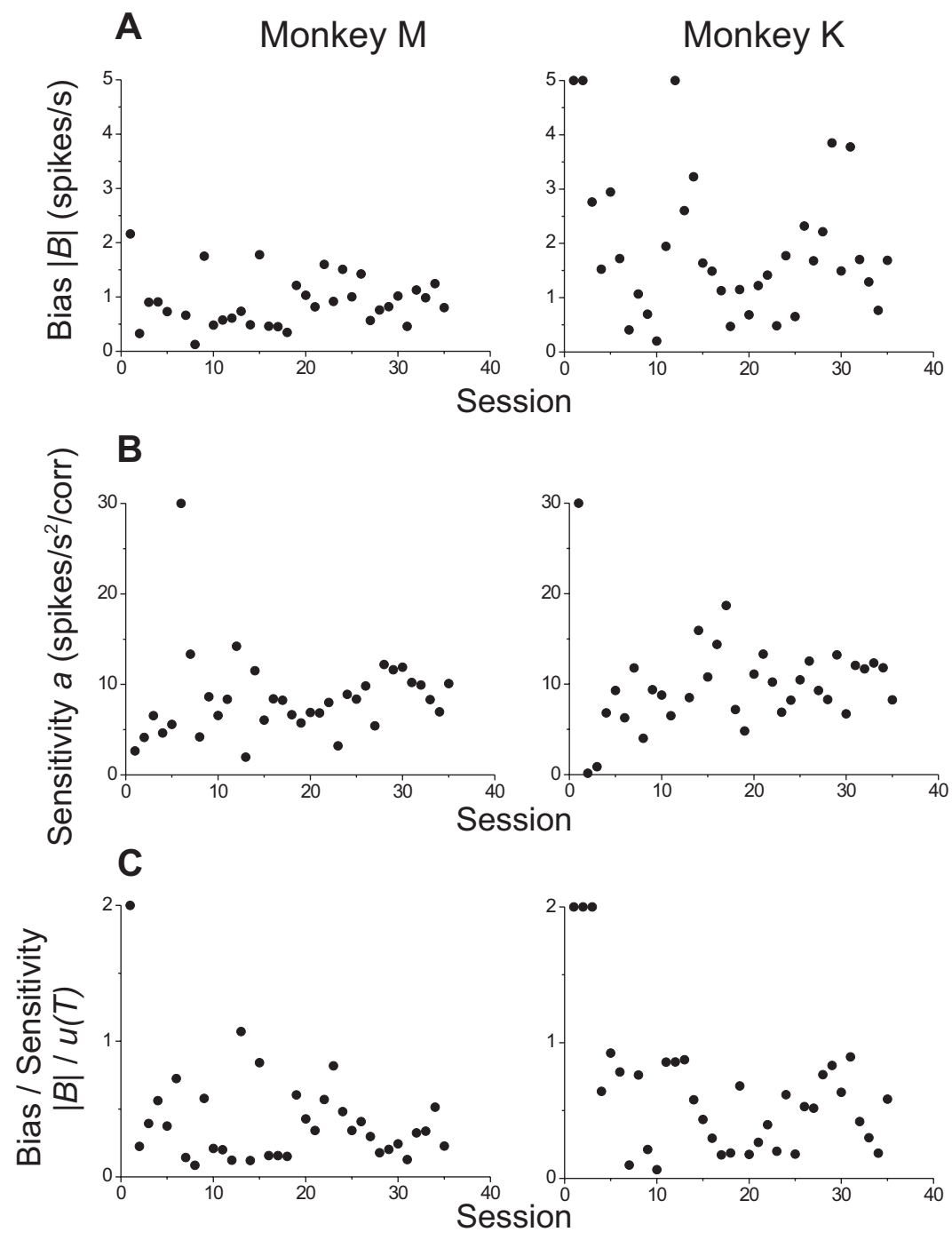

Figure 6. Bias analysis for the transfer experiment. $\boldsymbol{A}$, The amount of bias is plotted as a function of recording sessions separately for the two monkeys during learning at new locations. Biases larger than 5 are plotted as 5 . B, Sensitivities are plotted as a function of recording sessions separately for the two monkeys during learning at new locations. Sensitivities larger than 30 are plotted as 30. C, The relative contributions of biases compared with sensory signals are plotted as a function of recording sessions separately for the two monkeys during learning at new locations. Values larger than 2 are plotted as 2.

\section{Improvement of psychophysical sensitivity due to learning}

We first examined how behavioral sensitivity changed due to learning. For this purpose, we calculated error rates at $96 \%$ correlation, the highest correlation that was used (Fig. 2B). These values can represent lapse rate and may provide a measure of how well the monkeys understood the task (Law and Gold, 2008). The monkeys' lapse rates decreased quickly, suggesting that task learning occurred quickly. We then exploited psychometric functions and fit them with a Weibull function to calculate psychophysical thresholds as the binocular correlation level necessary to achieve $82 \%$ correct performance. To determine how behavioral performance improved due to training, we examined how psychophysical thresholds changed across daily recording sessions. Figure $2 B$ shows changes in psychophysical thresholds for the two monkeys. Because the largest binocular correlation used was always $96 \%$, thresholds $>96 \%$ were unreliable. Therefore, thresholds $>96 \%$ are plotted as $200 \%$ in the figures. In general, psychophysical thresholds decreased as recording sessions progressed, reaching a plateau during the latter half of the recording sessions. We quantified the long-term improvement rate of lapse rate and psychophysical threshold by fitting the curves in Figure $2 B$ with an exponential function and determining the time constants (Law and Gold, 2008). Because thresholds larger than $96 \%$ were unreliable, we estimated the time constants in two ways, one excluding thresholds $>96 \%$ and another using all the data. Time constants for lapse rate were smaller than those for psychophysical threshold, suggesting that task learning occurred faster than perceptual learning (Table 1). We further determined a nonparametric time constant (see Materials and Methods, above). The nonparametric time constants were smaller for lapse rates compared with thresholds (Table 1), further confirming that task learning occurred faster than perceptual learning. Overall, the median psychophysical threshold calculated from the last 10 sessions was $25.9 \%$ for monkey $\mathrm{G}$ and $43.8 \%$ for monkey P. Although the thresholds for monkey $\mathrm{P}$ were higher than expected, the thresholds for monkey G were in line with those reported from a previous study using two well trained monkeys [median psychophysical threshold $=20.9 \%$ and $29.2 \%$ (Uka and DeAngelis, 2003)].

It is important to determine whether the observed decrease in thresholds was due to improvements in eliminating unnecessary behavioral biases or to genuine improvements in sensitivity. We therefore analyzed how biases affected learning. For each session, we first estimated the amount of bias by calculating sequential biases (i.e., biases based on previous choices and reward), and then implemented them in the psychometric function (see Materials and Methods, above) (Gold et al., 2008). This allowed us to calculate sensitivity taking out the possible influence of bias. The estimated amount of bias decreased with session for both monkeys (Spearman's $r=-0.26, p=0.028, n=70$ for monkey $\mathrm{G}, r=$ $-0.29, p=0.0038, n=98$ for monkey P; Fig. $3 A$ ). Sensitivity to depth increased with session for both monkeys (Spearman's $r=$ $0.44, p=0.0002, n=70$ for monkey G, $r=0.59, p<0.0001, n=$ 98 for monkey P; Fig. $3 B$ ), and consequently, the relative contribution of biases compared with the dependence on sensory signals decreased with session (Fig. 3C). Importantly, sensitivity was calculated taking out the effects of biases. Even in this case, the decrease in bias occurred earlier than the increase in sensitivity. This implies that task learning occurred quickly, and that the observed decrease in psychophysical thresholds was most likely due, at least in part, to genuine improvements in behavioral sensitivity.

\section{No change in neuronal sensitivity due to learning}

To determine whether the long-term improvements in psychophysical sensitivity observed in our monkeys were accompanied by improvements in neuronal sensitivity in area MT, we recorded 
from isolated MT neurons while the monkeys learned the depth-discrimination task. Each day, a neuron was isolated and its receptive field and tuning properties were mapped quantitatively. Discrimination trials then commenced with the stimulus properties tailored to the tuning properties of the neuron.

We first calculated neuronal thresholds using ROC analysis (Britten et al., 1992; Uka and DeAngelis, 2003). Not surprisingly, neuronal thresholds were small; thus, neurons were sensitive to binocular correlation, even at the beginning of training (Fig. $4 A$; thresholds $>96 \%$ are shown as 200\%). For both monkeys, neuronal thresholds were $<30 \%$ for the first three sessions, and no correlation was observed between neuronal threshold and recording session (Spearman's $r=0.068$, $p=0.57, n=70$ for monkey $\mathrm{G} ; r=0.038$, $p=0.71, n=98$ for monkey P). Overall, median neuronal thresholds were $23.2 \%$ and $25.0 \%$ for monkeys $\mathrm{G}$ and $\mathrm{P}$, respectively, which is in line with thresholds previously reported using two well trained monkeys [median neuronal threshold = 21.3\% and 23.9\% (Uka and DeAngelis, 2003)].

At the beginning of training, psychophysical thresholds were almost always larger than neuronal thresholds. Thus, neuronal to psychophysical threshold ratios (NP ratios) were almost always less than one at the beginning of training (Fig. $4 B$ ). The NP ratio then started to rise with training; psychophysical thresholds decreased toward neuronal thresholds, bringing the NP ratio close to 1 . Here, NP ratios were plotted for sessions where both psychophysical and neuronal thresholds were smaller than 96\%. Thus, NP ratios were not shown early during training, but they obviously would have been much lower than 1 if psychophysical thresholds were measurable. Overall, long-term improvements in psychophysical sensitivity were not accompanied by improvements in neuronal sensitivity in area MT. Rather, processing beyond MT might change so that information within sensitive MT neurons that existed from the beginning of training is effectively read out for decisions about depth.

\section{Long-term rise of choice-related response modulation due to learning}

If processing beyond MT is altered due to training, we might be able to observe a signature of this change by inspecting the relationship between MT responses and the decision that the monkey made. We thus measured the degree of choice-related response modulation by calculating $\mathrm{CP}$. CP refers to the probability that one could predict the monkey's choice from the responses of sensory neurons (Britten et al., 1996; Uka and DeAngelis, 2004). $\mathrm{CP}$ is known to be $>0.5$ during the depth-discrimination task for MT neurons in well trained monkeys (Uka and DeAngelis, 2004). We therefore expected CP to increase during depth-discrimination task learning.

Figure $4 C$ shows $\mathrm{CP}$ data from the two monkeys. CP indeed increased with training for both monkeys. CP first hovered around 0.5 and then increased as recording sessions progressed, although the degree of increase differed markedly between the two monkeys. For monkey G, CP was high after $\sim 15$ sessions and reached a plateau of $\sim 0.68$ (median of the last 20 sessions) after 30 sessions. CP was even $>0.9$ for some neurons. For monkey $\mathrm{P}, \mathrm{CP}$ only increased slightly after $>50$ sessions, and the plateau was $\sim 0.57$. Although we do not know why the magnitude of CP differed between monkeys, the correlation between $\mathrm{CP}$ and recording session was significant for both monkeys (Spearman's $r=0.30, p=0.013, n=70$ for monkey $\mathrm{G} ; r=0.33, p=0.0011 n=98$ for monkey $\mathrm{P}$ ).

An important point is whether the rise in $\mathrm{CP}$ is due to task learning or perceptual learning. To address this question, we asked whether CP is correlated with lapse rate or psychophysical thresholds. Because lapse rate and psychophysical thresholds are correlated with each other, we calculated the partial correlation between $\mathrm{CP}$ and both measures. Additionally, because $\mathrm{CP}$ is known to be correlated with neuronal thresholds (Britten et al., 1996; Uka and DeAngelis, 2004), we added neuronal thresholds as a factor as well. Partial correlations were calculated, including thresholds larger than $96 \%$. Significant negative partial correlations were observed between lapse rate and CP $(r=-0.39, p<$ 0.001, $n=70$ for monkey G; $r=-0.20, p=0.047, n=98$ for monkey P), but not between psychophysical threshold and CP for both monkeys ( $r=0.20, p=0.094, n=70$ for monkey G; $r=$ $-0.01, p=0.87, n=98$ for monkey $\mathrm{P}$ ). This was true even if the correlation analyses after calculation of residuals were nonparametric (residuals lapse rate vs residual CP; Spearman's $r=-0.26$, $p=0.032, n=70$ for monkey G; $r=-0.22, p=0.032, n=98$ for monkey P: residual threshold vs residual CP; Spearman's $r=$ $0.15, p=0.22, n=70$ for monkey G; $r=0.16, p=0.12, n=98$ for monkey $\mathrm{P}$ ). Thus, the overall increase in $\mathrm{CP}$ seems to be restricted to early phases of learning. 


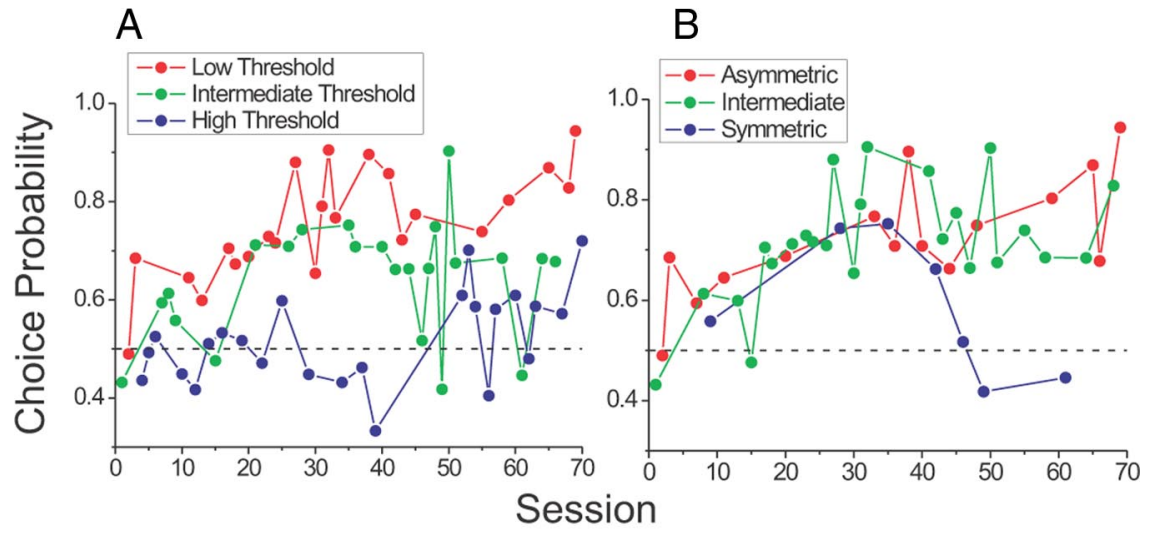

C

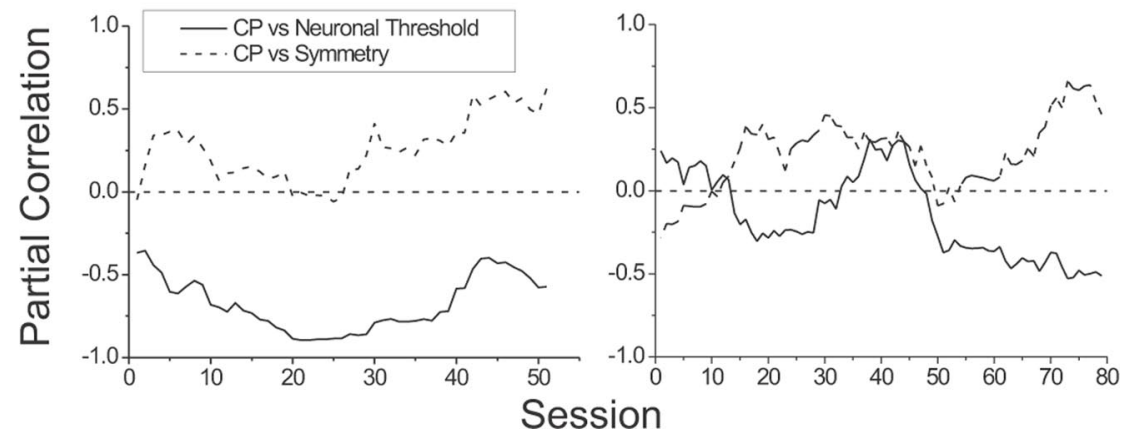

Figure 8. Relationship between CP and neuronal threshold/disparity-tuning symmetry. $A, C P$ data were divided into three groups depending on neuronal threshold for monkey $G$. CP is plotted against recording sessions separately for the three groups. $\boldsymbol{B}$, $C P$ data were divided into three groups depending on disparity-tuning symmetry for monkey $G$. $C P$ is plotted against recording sessions separately for the three groups. Neurons with high thresholds were excluded. C, Partial correlation between CP and neuronal thresholds (filled circles) or (P and disparity-tuning symmetry (open circles) are plotted against recording sessions. Data were grouped into 20-session windows, and partial correlations were calculated within each window. Session corresponds to the beginning of the 20-session window.

\section{Transfer across visual field}

A signature of perceptual learning is that, in many cases, learning does not transfer across stimulus parameters, particularly across visual field (Schoups et al., 1995). Recent studies, however, have shown considerable transfer of learning across stimulus parameters (Jeter et al., 2010; Zhang et al., 2010). Because the outcome of transfer experiments has led to different views concerning the neuronal mechanism underlying perceptual learning, we thought it important to measure both psychophysical and neuronal transfer in our experimental setup. To explicitly test how behavioral sensitivity, as well as CP magnitude, transferred across visual fields, we trained two more monkeys on the depth-discrimination task at a fixed location (Fig. $5 \mathrm{~A}$, black circle) before recording from MT neurons in the ipsilateral hemisphere (i.e., receptive fields were in the opposite hemifield). As we wanted the monkeys to generalize their learning across different stimulus parameters but not across different spatial locations, for each training session, groups of motion direction, speed, and binocular disparities were randomly chosen from parameters used in recording sessions from the previous two monkeys. Figure $5 B$ shows that both lapse rate and psychophysical thresholds decreased with training at the fixed location, although it took longer for monkey $\mathrm{M}$ to reach a plateau in psychophysical performance compared with other monkeys. A total of 192 training sessions, consisting of 107,520 total trials, and 100 training sessions, consisting of 56,000 total trials, were performed for monkey $\mathrm{M}$ and monkey $\mathrm{K}$, respectively. Time constants from exponential fits confirmed that task learning occurred faster than perceptual learning in both monkeys (Table 1).

After training at the fixed location, we recorded from MT neurons. Figure $5 A$ (red circles) shows the receptive field location of the recorded neurons relative to the fixed training location in the opposite hemifield. Receptive fields did not overlap with the trained location. We next evaluated whether behavioral learning of the depth-discrimination task at the fixed location transferred across visual fields. Red triangles and circles in Figure $5 C$ show lapse rate and psychophysical thresholds, respectively, while neurons were being recorded simultaneously. Both lapse rate and psychophysical thresholds slightly decreased as recording sessions progressed. In addition, learning was faster in the transfer hemifield compared with initial training, as was confirmed by the time constant of exponential fits (Table 1). This shows that although learning was specific to the trained hemifield, generalization of learning that enabled fast learning in the untrained hemifield occurred across visual fields.

Because stimulus parameters were always tailored to the neuron's preference, parameters such as location, motion direction, speed, and binocular disparity were different for each recording session. This could have induced variability in psychophysical performance (Uka and DeAngelis, 2003) and hampered our behavioral-improvement measurement. Therefore, on the day following each recording session, the monkeys performed the depth-discrimination task at the previously trained location (Fig. 5A, black circle) using identical parameters except spatial location and aperture size. Psychophysical thresholds from those sessions are shown as black circles in Figure $5 C$. The threshold ratio (untrained/trained location) shown in Figure $5 D$ was then used as a measure of improvement in behavior in the nontrained hemifield. The threshold ratio gradually decreased, and a negative correlation between threshold ratio and recording session (Spearman's $r=-0.57, p<0.001, n=35$ for monkey M; $r=-0.32$, $p=0.068, n=35$ for monkey K) indicated that the monkeys' performance improved with training. This confirms that learning did indeed occur in the untrained hemifield.

We also recalculated the psychometric function taking bias into account (Fig. 6). During recording, bias did not decrease (Spearman's $r=0.15, p=0.40, n=35$ for monkey M; $r=-0.13, p=0.45$, $n=35$ for monkey K; Fig. 6 A), but rather stayed stable. Additionally, sensitivity (taking out the effect of bias) slightly increased (Spearman's $r=0.37, p=0.029, n=35$ for monkey M; $r=0.23, p=0.18$, $n=35$ for monkey K; Fig. $6 B$ ). Thus, the ability to attenuate biases, presumably reflecting the knowledge that discrimination should be based on sensory signals and not on the temporal structure of choice and reward (task learning), transferred across visual field. Modest learning also occurred at the untrained location.

Given the specificity and generalization of learning across visual fields, we analyzed how neuronal characteristics transferred across visual fields. Figure $7 A$ shows neuronal thresholds across recording 
sessions. As was expected from the initial experiments described above, neurons with low thresholds were observed in the early phase of recording sessions, and no correlation between neuronal thresholds and recording sessions was found (Spearman's $r=$ $-0.10, p=0.55, n=35$ for monkey M; $r=$ $-0.19, p=0.27, n=35$ for monkey $\mathrm{K})$. A more interesting question is how $\mathrm{CP}$ behaved during the course of the recording session. Interestingly, CP did not increase during the course of the recording session, but had a rather high value from the beginning (Fig. 7B). CP was over 0.6 for seven and four of the first 10 recording sessions for monkey $\mathrm{M}$ and monkey $\mathrm{K}$, respectively, and no correlation between $\mathrm{CP}$ and recording session was observed (Spearman's $r=-0.061, p=0.73, n=35$ for monkey $\mathrm{M} ; r=-0.10, p=0.57, n=35$ for monkey $\mathrm{K}$ ). The median $\mathrm{CP}$ was 0.61 and 0.54 across all recording sessions for monkey $\mathrm{M}$ and monkey $\mathrm{K}$, respectively, which was significantly greater than 0.5 ( sign test, $p=0.006, n=35$ for monkey M; $p=0.009, n=35$ for monkey K). Thus, even though residual learning occurred in the newly trained visual field, this was not accompanied by an increase in CP.

Overall, our transfer experiment showed both specificity and generalization of learning across visual fields. Psychophysical performance in the untrained location was initially worse than that in the trained hemifield, but learning was faster compared with initial training. Additionally, we found no changes in neuronal characteristics. This suggests that changes in the relationship between MT responses and behavioral choices are restricted to initial task learning.

\section{Increase in choice-related response modulation depends on neuronal sensitivity and disparity-tuning symmetry}

$\mathrm{CP}$ is known to correlate with neuronal sensitivity and disparitytuning symmetry; neurons with smaller thresholds and those that have peaks and troughs on opposite sides of zero disparity (i.e., asymmetric disparity tuning) tend to have a larger CP (Uka and DeAngelis, 2004). Here, we analyzed how this relationship developed over the time course of learning.

Figure $8 \mathrm{~A}$ shows $\mathrm{CP}$ data for neurons divided into three groups depending on neuronal threshold for monkey G. For lowthreshold neurons, CP rose quickly and little thereafter; CP for neurons with intermediate thresholds rose as well. CP for highthreshold neurons was never large. Figure $8 B$ shows $C P$ data divided into three groups depending on tuning symmetry for monkey G. In this figure, high-threshold neurons were excluded because they never showed high CP. Tuning symmetry was calculated by fitting a Gabor function (with the center of the Gaussian fixed at zero) to the disparity-tuning curve and extracting the phase parameter (Uka and DeAngelis, 2004). Interestingly, CP for both symmetric and asymmetric neurons rose early during training, but CP for symmetric neurons dropped late in training.

To quantify these impressions, we calculated how the correlation between $\mathrm{CP}$ and neuronal threshold/tuning symmetry developed across training sessions. Data were grouped into 20session windows, and the window slid every session, while partial correlations between CP and neuronal thresholds or CP and tun- ing symmetry were calculated within each window. The running partial correlations are plotted in Figure $8 C$. Solid lines denote the partial correlation between $\mathrm{CP}$ and neuronal thresholds. A negative correlation indicates that neurons with smaller thresholds (i.e., the more sensitive neurons) had a larger CP. For monkey G, a negative correlation was apparent from the beginning, strengthened for the first 20 sessions, and reached a plateau thereafter. For monkey P, the negative correlation was apparent only after 50 sessions. Broken lines denote the partial correlation between $\mathrm{CP}$ and tuning symmetry. A positive correlation indicates that neurons with asymmetric tuning (i.e., neurons with a peak and trough at opposite sides of zero) had a larger CP. For monkey $\mathrm{G}$, a positive correlation gradually developed after 30 sessions and reached a plateau thereafter. For monkey P, the positive correlation gradually developed after 70 sessions. Thus, a negative correlation between $\mathrm{CP}$ and neuronal thresholds and a positive correlation between CP and tuning symmetry were observed in both monkeys. It also seems that the correlation between CP and neuronal thresholds developed earlier than the correlation between CP and tuning symmetry, suggesting that the monkeys first learned to read out signals from the more sensitive neurons and later learned to fine tune their strategy to read out signals from asymmetrically tuned neurons.

\section{Comparison with human learning}

In theory, it is difficult to dissociate task learning with perceptual learning. For descriptive purposes, we associated changes in lapse rate with task learning and changes in psychophysical thresholds with perceptual learning. We further incorporated biases into the psychometric function. Decrease in bias may be associated with task learning, and increase in sensitivity with genuine perceptual learning (see Discussion, below). Although these are convenient ways to separate the two, it is not clear whether results obtained in the present study are comparable to those described in the human psychophysical literature. To address this question, we conducted the same psychophysical experiments in two male human subjects and compared them with the monkeys' results. The subjects were given explicit instruction to discriminate near versus far. One of the subjects did not learn: psychophysical thresholds were fairly low from the beginning and did not improve with 
training sessions. The other subject had a learning curve that was very similar to that of the monkey. Data for this subject is shown in Figure 9. Learning rates calculated from the exponential fits are shown in Table 1, although the time constant for psychophysical thresholds excluding thresholds larger than $96 \%$ in the untrained hemifield is misleading because the subject's threshold was $>96 \%$ on the second day, and thus not included in the exponential fit. Nonetheless, although there seems to be considerable individual difference, both in humans and monkeys, learning rates overlap with each other. Moreover, learning was faster at the untrained location. We further incorporated biases into the psychometric function. Bias did not decrease with session either during initial learning or in the untrained location in the transfer experiment (Spearman's $r=-0.038, p=0.79, n=50$ for initial learning; $r=0.045, p=0.83, n=25$ for untrained location: transfer experiment). Sensitivity changes were observed during initial training and modestly at the untrained location in the transfer experiment (Spearman's $r=0.75, p<0.0001, n=50$ for initial learning; $r=0.10, p=0.63, n=25$ for untrained location: transfer experiment). This suggests that long-term changes in the structure of biases were different between monkeys and humans during initial learning, presumably due to explicit instructions given to the human. The occurrence of fast and modest learning at the transfer location was comparable between monkeys and humans.

\section{Discussion}

In this study, we first recorded from isolated neurons in area MT while monkeys learned to perform a depth-discrimination task. Long-term improvement in behavioral performance was accompanied by an increase in the correlation between MT responses and behavioral choice (CP), although neuronal sensitivity did not change due to learning. The increase in $\mathrm{CP}$ was correlated with improvements in lapse rate. Our interpretation is that long-term plastic changes occur beyond the processing in MT, such that the readout of information from MT improves due to task learning. We further explicitly tested whether behavioral learning and changes in neuronal properties transferred across visual fields. We found that although behavioral performance in the untrained hemifield was initially worse than that in the trained hemifield, learning was faster compared with initial training. Furthermore, $\mathrm{CP}$ was high from the beginning of training in the untrained visual field. We propose that the basic functional connections between MT neurons and those mediating eye movements change in both hemispheres during initial task learning, enabling fast learning in the untrained hemifield. Changes underlying learning in the untrained hemifield presumably are mediated by mechanisms that are not detected by the magnitude of $\mathrm{CP}$, although structural changes in $\mathrm{CP}$, such as correlations between $\mathrm{CP}$ and disparity tuning symmetry, can occur without an increase in the mean magnitude of CP.

\section{Task learning and perceptual learning}

What exactly is learned during learning of a perceptual task? We hypothesized that monkeys initially tried to figure out the temporal structure of choice and reward, which led to specific sequential biases. Sequential biases decreased during initial learning, suggesting that the monkeys initially adopted a strategy to search for temporal structure of choice and reward but later concentrated on the relationship between sensory signal and choice. In the transfer experiment, biases did not decrease with session, implying that the monkeys knew they were to rely on sensory signals: i.e., task learning transferred across visual field.
Even in this case, sensitivity to depth increased, albeit modestly, with session, suggesting that learning unrelated to task rule learning occurred. In one human subject whose learning curve was similar to the monkeys, biases did not decrease with session during initial learning. This is understandable because, based on the explicit instructions, the subject knew he had to rely on sensory signals and not on the temporal structure of choice and reward. Fast learning at the untrained location also occurred in the human subject during the transfer experiment. Together, we believe that task learning in monkeys was reflected in the decrease in bias across sessions during initial learning. Improvements of sensitivity with constant biases presumably correspond to genuine perceptual learning, which was observed in both human and monkey. Fast learning at an untrained location may, in part, be due to stable biases during the transfer experiment for monkeys, but the occurrence in human suggests the functioning of alternative mechanisms.

\section{Implication for long-term learning}

Perceptual learning is often visual-field-specific, which has led to the view that learning occurs in early visual areas that contain neurons with small receptive fields (Karni and Sagi, 1991). Indeed, several investigators have found an increase in the sensitivity of sensory neurons in both areas V1 (Schoups et al., 2001) and V4 (Yang and Maunsell, 2004; Raiguel et al., 2006) of the macaque monkey. However, the change in sensitivity is very small, and conflicting reports have been made as well. Ghose et al. (2002) used a similar orientation-discrimination task as Schoups et al. (2001), but found no increase in the sensitivity of V1 neurons. Thus, an increase in the sensitivity of sensory neurons is unlikely to explain the magnitude of learning observed behaviorally.

Recently, Law and Gold (2008) recorded from neurons in areas MT and LIP during long-term learning of a motiondirection discrimination task. They showed that although sensitivity of MT neurons to motion direction did not change with training, the rate of build-up activity in LIP increased. They also showed that the relationship between neuronal sensitivity and CP became stronger with learning. Law and Gold (2008) interpreted their results as evidence that the readout of MT signals changes during training.

Our results from the two monkeys recorded from initial training are basically in line with Law and Gold (2008), complementing them using a similar but different task. It is clear that sensitive neurons exist from the beginning of training. Moreover, CP increased during initial learning. The increase in $\mathrm{CP}$, however, was restricted to early phases of training when monkeys were learning the task. Our transfer experiment further showed that CP was large from the beginning of training in the untrained hemifield. This indicates that a large CP can be observed without previous training of the task at that particular location.

There are at least two interpretations for these results. First, CP could reflect changes in noise correlation (Shadlen et al., 1996; Nienborg and Cumming, 2010). Indeed, recent studies have found that the structure of noise correlation is not fixed, but is dynamic (Cohen and Newsome, 2008; Cohen and Maunsell, 2009; Mitchell et al., 2009). In this case, the transfer experiment implies that noise correlation structure changes in both hemispheres simultaneously during initial training, so that noise is more correlated within each MT after learning. An increase in noise correlation predicts, however, that the pooled MT response becomes less sensitive, which contradicts the improved behavioral sensitivity. Second, a large CP may be an indication that a neuron is more dedicated to the decision process (Uka and 
DeAngelis, 2004; Law and Gold, 2008). Indeed, Gu et al. (2011) showed that variations in $\mathrm{CP}$ were not explained by the structure of noise correlation among nearby neurons, giving support for this theory. In this case, readout from MT neurons should change in both hemispheres simultaneously during initial training. It is also possible that the structure underlying MT and the decision process does not change during learning, but the relative contribution of MT to decision increases due to reduction in biases.

Our transfer experiment further showed that learning in the untrained hemifield was faster than initial learning. We propose that initial task learning shaped circuitry downstream from MT in both hemispheres, enabling fast learning in the untrained hemifield. This is consistent with recent psychophysical studies showing that task learning followed by exposure to visual stimulus enables transfer of perceptual learning (Zhang et al., 2010). Generalization of learning of this type is difficult to explain in a strict reinforcement learning framework (Sutton and Barto, 1998; Law and Gold, 2009) or with the Hebbian rule (Hebb, 1949) because MT neurons in the untrained hemifield were not activated. Hence, studies aimed to understand the mechanism underlying generalization and additional learning in the untrained visual field merits future investigation.

\section{References}

Britten KH, Shadlen MN, Newsome WT, Movshon JA (1992) The analysis of visual motion: a comparison of neuronal and psychophysical performance. J Neurosci 12:4745-4765.

Britten KH, Newsome WT, Shadlen MN, Celebrini S, Movshon JA (1996) A relationship between behavioral choice and the visual responses of neurons in macaque MT. Vis Neurosci 13:87-100.

Chowdhury SA, DeAngelis GC (2008) Fine discrimination training alters the causal contribution of macaque area MT to depth perception. Neuron 60:367-377.

Cohen MR, Maunsell JH (2009) Attention improves performance primarily by reducing interneuronal correlations. Nat Neurosci 12:1594-1600.

Cohen MR, Newsome WT (2008) Context-dependent changes in functional circuitry in visual area MT. Neuron 60:162-173.

DeAngelis GC, Uka T (2003) Coding of horizontal disparity and velocity by MT neurons in the alert macaque. J Neurophysiol 89:1094-1111.

DeAngelis GC, Cumming BG, Newsome WT (1998) Cortical area MT and the perception of stereoscopic depth. Nature 394:677-680.

Dosher BA, Lu ZL (1999) Mechanisms of perceptual learning. Vision Res 39:3197-3221.

Eckhoff P, Holmes P, Law C, Connolly PM, Gold JI (2008) On diffusion processes with variable drift rates as models for decision making during learning. New J Phys 10: nihpa49499.

Fiorentini A, Berardi N (1980) Perceptual learning specific for orientation and spatial frequency. Nature 287:43-44.

Ghose GM, Yang T, Maunsell JH (2002) Physiological correlates of perceptual learning in monkey V1 and V2. J Neurophysiol 87:1867-1888.

Gold JI, Law CT, Connolly P, Bennur S (2008) The relative influences of priors and sensory evidence on an oculomotor decision variable during perceptual learning. J Neurophysiol 100:2653-2668.

Gu Y, Liu S, Fetsch CR, Yang Y, Fok S, Sunkara A, DeAngelis GC, Angelaki DE (2011) Perceptual learning reduces interneuronal correlations in macaque visual cortex. Neuron 71:750-761.
Hebb DO (1949) The organization of behavior. New York: Wiley.

Jacobs RA (2009) Adaptive precision pooling of model neuron activities predicts the efficiency of human visual learning. J Vis 9:22.1-22.15.

Jeter PE, Dosher BA, Liu SH, Lu ZL (2010) Specificity of perceptual learning increases with increased training. Vision Res 50:1928-1940.

Judge SJ, Richmond BJ, Chu FC (1980) Implantation of magnetic search coils for measurement of eye position: an improved method. Vision Res 20:535-538.

Karni A, Sagi D (1991) Where practice makes perfect in texture discrimination: evidence for primary visual cortex plasticity. Proc Natl Acad Sci U S A 88:4966-4970.

Law CT, Gold JI (2008) Neural correlates of perceptual learning in a sensory-motor, but not a sensory, cortical area. Nat Neurosci 11:505-513.

Law CT, Gold JI (2009) Reinforcement learning can account for associative and perceptual learning on a visual-decision task. Nat Neurosci 12:655-663.

Maunsell JH, Van Essen DC (1983) Functional properties of neurons in middle temporal visual area of the macaque monkey. II. Binocular interactions and sensitivity to binocular disparity. J Neurophysiol 49:1148-1167.

Michel MM, Jacobs RA (2008) Learning optimal integration of arbitrary features in a perceptual discrimination task. J Vis 8:3.1-3.16.

Mitchell JF, Sundberg KA, Reynolds JH (2009) Spatial attention decorrelates intrinsic activity fluctuations in macaque area V4. Neuron 63:879-888.

Mollon JD, Danilova MV (1996) Three remarks on perceptual learning. Spat Vis 10:51-58.

Nienborg H, Cumming B (2010) Correlations between the activity of sensory neurons and behavior: how much do they tell us about a neuron's causality? Curr Opin Neurobiol 20:376-381.

Petrov A, Dosher BA, Lu ZL (2005) The dynamics of perceptual learning: an incremental reweighting model. Psychol Rev 112:715-743.

Raiguel S, Vogels R, Mysore SG, Orban GA (2006) Learning to see the difference specifically alters the most informative V4 neurons. J Neurosci 26:6589-6602

Schoups AA, Vogels R, Orban GA (1995) Human perceptual learning in identifying the oblique orientation: retinotopy, orientation specificity and monocularity. J Physiol 483:797-810.

Schoups AA, Vogels R, Qian N, Orban GA (2001) Practicing orientation identification improves orientation coding in V1 neurons. Nature 412:549-553.

Shadlen MN, Britten KH, Newsome WT, Movshon JA (1996) A computational analysis of the relationship between neuronal and behavioral responses to visual motion. J Neurosci 16:1486-1510.

Sutton RS, Barto AG (1998) Reinforcement learning. Cambridge, MA: MIT.

Uka T, DeAngelis GC (2003) Contribution of middle temporal area to coarse depth discrimination: comparison of neuronal and psychophysical sensitivity. J Neurosci 23:3515-3530.

Uka T, DeAngelis GC (2004) Contribution of area MT to stereoscopic depth perception: choice-related response modulations reflect task strategy. Neuron 42:297-310.

Uka T, DeAngelis GC (2006) Linking neural representation to function in stereoscopic depth perception: roles of the middle temporal area in coarse versus fine disparity discrimination. J Neurosci 26:6791-6802.

Yang T, Maunsell JH (2004) The effect of perceptual learning on neuronal responses in monkey visual area V4. J Neurosci 24:1617-1626.

Zhang JY, Zhang GL, Xiao LQ, Klein SA, Levi DM, Yu C (2010) Rule-based learning explains visual perceptual learning and its specificity and transfer. J Neurosci 30:12323-12328. 